\title{
TOT ambulatorio con ajuste intraoperatorio de la malla en pacientes con incontinencia urinaria de esfuerzo
}

\author{
Francisco Kaplan D. ${ }^{1}$, Robinson Flores C. ${ }^{1}$, Hernán Quinchavil A. ${ }^{1}$, Oscar \\ Becerra A. 1, Tiare Hevia G. a, Cristián Brito Y. b \\ ${ }^{1}$ Servicio de Obstetricia y Ginecología, Hospital de Quilpué, 5ª Región, Chile. \\ a Matrona, Hospital de Quilpué. \\ b Alumno, Facultad de Medicina, Universidad Andrés Bello.
}

\section{RESUMEN}

Antecedentes: La Incontinencia Urinaria de Esfuerzo (IUE) es una patología frecuente y en ascenso entre las mujeres, produce un deterioro significativo en la calidad de vida de ellas. Objetivos: Evaluar los resultados en el tratamiento de la IUE mediante un TOT ambulatorio con ajuste intraoperatorio de la tensión de la malla. Método: Se realizó un estudio prospectivo, descriptivo en el que se evaluaron los resultados objetivos y subjetivos en el tratamiento de la IUE con TOT ambulatorio, con ajuste intraoperatorio de la tensión de la malla. Resultados: Se obtuvo continencia normal en el $93,3 \%$ de las pacientes, $87 \%$ se consideró "sana". No se presentaron complicaciones mayores ni sobrecorrección a los 24 meses de seguimiento. El 93\% de las pacientes abandonó el hospital el mismo día de la cirugía. Conclusiones: El ajuste de la malla intraoperatorio permitiría mejorar los resultados publicados de continencia en la cirugía de TOT ambulatorio, disminuyendo el riesgo de sobrecorrección de la IUE en forma segura y rápida. Consideramos que debiera ser un paso obligatorio en el TOT.

\section{PALABRAS CLAVE: TOT, ajuste intraoperatorio de la malla, incontinencia de orina}

\section{SUMMARY}

Background: Stress urinary incontinence (SUI) is a common condition and rising among women, with a significant deterioration of quality of life. Objectives: To evaluate the results in the treatment of SUI by ambulatory TOT with intraoperative adjustment of mesh tension. Methods: We performed a prospective descriptive study that evaluated the objective and subjective outcomes of the SUI treatment with ambulatory TOT intraoperative adjustment of mesh tension. Results: Normal continence was obtained $93.3 \%$ of patients, $87 \%$ were considered cured. There were no major complications or over correction at 24 months follow up. $93.3 \%$ were discharged on the day of surgery. Conclusions: The intraoperative adjustment of the mesh would improve continence results published in TOT outpatient surgery, reducing the risk of overcorrection of SUI safely and quickly. We believe it should be a mandatory step in the TOT.

KEY WORDS: TOT, intraoperative adjustment of mesh, urinary incontinence 


\section{INTRODUCCIÓN}

La incontinencia urinaria (IU) se define como la pérdida involuntaria de orina. Es considerada como un problema médico y social en ascenso, a causa del progresivo envejecimiento de la población. Aunque no se trata de un proceso de riesgo vital produce un deterioro significativo en la calidad de vida de las personas, limitando su autonomía y reduciendo su autoestima.

La IU afecta a un gran número de personas en el mundo, la mayoría de ellas son mujeres. Paradojalmente, sólo el $25 \%$ de ellas pide ayuda médica, debido a consideraciones sociales, culturales o personales. La IU tiene una prevalencia de 10 a $30 \%$ en mujeres entre los 15 y 64 años y de 10 a $40 \%$ en las mujeres mayores de 64 años. Hay distintos tipos de IU, las más frecuentes son: la incontinencia urinaria de esfuerzo (IUE), la incontinencia urinaria por urgencia (IUU) y la incontinencia urinaria mixta (IUM). En mujeres menores de 60 años la IUE representa el $58 \%$ de las IU y en las mujeres mayores de 60 años el 36\% (1).

La IUE se define como la pérdida involuntaria de orina con los esfuerzos, ejercicio, estornudo o tos, se produce cuando la presión intravesical sobrepasa a la uretral, secundaria a la hipermovilidad uretral, al daño intrínseco del esfínter uretral o ambos (2). Actualmente existe una teoría complementaria a las anteriores, que sugiere que la IUE se produciría por una incompetencia transitoria de mecanismos funcionales de la continencia, secundarios a alteraciones anatómicas al esfuerzo, estos mecanismos se bloquean con la anestesia raquídea permitiendo, en mujeres sanas, escape de orina con presiones intravesicales mayores a 30 - 40 $\mathrm{cm} \mathrm{H} 2 \mathrm{O}$ (3).

Las mallas a nivel de la uretra media han cambiado el manejo actual de la IUE permitiendo un tratamiento quirúrgico eficaz, mínimamente invasivo y en el caso de la malla transobturatriz, con mínimo riesgo de complicaciones mayores (4).

El objetivo de este estudio es evaluar el resultado de la continencia urinaria y las complicaciones en el tratamiento quirúrgico de la IUE mediante la técnica de TOT ambulatoria con ajuste intraoperatorio de la malla.

\section{PACIENTES Y MÉTODO}

Se realizó un estudio prospectivo, descriptivo, en el que se evaluó el resultado objetivo, el resultado subjetivo y las complicaciones en el tratamiento quirúrgico de la IUE, mediante la técnica de TOT ambulatorio con ajuste intraoperatorio de la tensión de la malla a $35 \mathrm{~cm}$ de $\mathrm{H} 2 \mathrm{O}$ con la vejiga llena, según su capacidad vesical (previamente cuantificada) (5), en el Hospital de Quilpué, 5ª Región, Chile, entre diciembre de 2008 y junio de 2011.

El diagnóstico de IUE se realizó por medio de una anamnesis detallada, cuestionario orientado a definir el tipo de IU, examen físico general y ginecológico, cartilla miccional, estudio cistométrico clínico (medición del residuo postmiccional, primer deseo miccional, capacidad vesical, presencia de contracciones vesicales anormales), test de estrés con vejiga llena positivo (escape de orina con maniobras de valsalva), prueba de TVT clínica positiva (imita digitalmente la contención suburetral en la zona media, similar a la que producirá la malla, permitiendo predecir el beneficio de la cirugía) (5).

Fueron incluidas en el estudio sólo aquellas pacientes que cumplían con todos los criterios diagnóstico de IUE pura con sus patologías asociadas, si existen, compensadas, exámenes de laboratorio normales (hemograma, glicemia, creatinemia, sedimento de orina, urocultivo y examen físico químico de orina), ecotomografía transvaginal normal y aprobación del consentimiento informado. Fueron excluidas aquellas pacientes con cirugías previas de IU, IUU, IUM, retención urinaria crónica, residuo postmiccional mayor a $50 \mathrm{ml}$, POP-Q mayor o igual a 2, patologías de base no compensadas, patologías ginecológicas quirúrgicas concomitantes y pacientes con desórdenes mentales.

Todas las pacientes ingresaron a la Unidad de Cirugía Ambulatoria del Hospital de Quilpué, fueron operadas por dos cirujanos y se utilizó una malla de polipropileno de $30 \times 1,5 \mathrm{~cm}$.

Técnica quirúrgica. Pacientes en posición ginecológica con anestesia raquídea (Bupivacaína). Asepsia perineal y vaginal con povidona yodada y vaciamiento vesical con sonda Nélaton de 12 FR. Incisión medio sagital de $2 \mathrm{~cm}$ de longitud de la pared vaginal anterior a 1,5 cm bajo el meato uretral, disección mínima de la mucosa vaginal con tijera hasta el borde inferior del tercio medio de la rama isquiopubiana de cada lado. Incisión cutánea de $5 \mathrm{~mm}$ en el pliegue inguinocrural de cada lado a nivel de línea imaginaria horizontal que pasa por el clítoris. Instalación de la malla de TOT por medio de ganchos de acero helicoidales fenestrados en su extremo distal, que perforan desde la incisión cutánea inguinocrural, pasando por el músculo aductor largo, grasilis, obturador externo, membrana obturatriz y obturador interno siguiendo el borde superior, posterior e inferior de la rama isquiopubiana, guiado por el dedo meñique del operador (para evitar disecciones extensas de la mucosa vaginal) hasta el exterior, por medio de la incisión medio sagital de la mucosa vaginal. Poste- 
riormente se llena la vejiga según la capacidad vesical y se ajusta la tensión de la malla hasta obtener escape urinario a una presión igual o mayor a $35 \mathrm{~cm}$ H2O medida con una sonda de alimentación de 6 FR, conectada a una columna de agua, mediante la compresión suprapúbica de la vejiga. Finalmente se seccionan los remanentes de malla de los pliegues inguinocrurales, se sutura la mucosa vaginal con catgut cromado no 2-0 y se extrae el suero de la vejiga con una sonda Nélaton de 12 FR.

En el postoperatorio se administran $500 \mathrm{ml}$ de suero fisiológico endovenoso en 3 horas y se realizan cateterismos vesicales cada 3 horas hasta que la paciente orine espontáneamente, con un residuo postmiccional menor de $100 \mathrm{ml}$ para el alta médica el mismo día de la cirugía. A todas las pacientes se les indica un óvulo de Metronidazol diario por 5 días e lbuprofeno de $400 \mathrm{mg}$ cada 8 horas por 2 días.

A todas las pacientes se les realizaron controles médicos post operatorio a las 6 horas, 7 días y 3,6 , 12,18 y 24 meses, que incluyeron anamnesis, encuesta orientada al diagnóstico de IUE y cualquier tipo de IU de novo, examen ginecológico, test de estrés con vejiga llena en decúbito dorsal y de pie y medición del residuo postmiccional. Por último se les realizó una encuesta subjetiva de satisfacción.

\section{RESULTADOS}

Ingresaron al estudio 17 pacientes de las cuales fueron eliminadas 2, por no seguir los controles. A todas las pacientes ( $n$ : 15$)$ se les hizo un seguimiento durante 24 meses.

El promedio de edad fue de 54 años (rango: 3570 años), IMC de 30 (rango: 21-42 kg/m2), partos normales 3 , cesáreas 1 y solo una paciente (1/15) tenía el antecedente de un parto fórceps. Ninguna paciente había sido histerectomizada y 2 presentaban prolapso de órganos pélvicos (POP) grado I según la clasificación de POP-Q.

La frecuencia promedio IUE semanal fue de 30 veces, residuo posmiccional (RPM) de $6,59 \mathrm{ml}$, primer deseo miccional de $180 \mathrm{ml}$, capacidad vesical de $426 \mathrm{ml}$. (Tabla I). En el 93,3\% (14/15) de las pacientes la estadía hospitalaria fue menor a 10 horas $y$ en el $100 \%(15 / 15)$ menor a 24 horas.

Todos los procedimientos cistométricos y quirúrgicos fueron realizados por dos cirujanos experimentados en la técnica. A todas las pacientes (n: 15) se les realizó un TOT con ajuste intraoperatorio de la malla. El 93,3\% (14/15) de las pacientes permanecieron objetivamente continentes a los 24 meses y sólo el 6,7\% (1/15) persistió con IUE, pero con una frecuencia significativamente menor (disminuyó de 70 veces semanal a 1 vez por semana). El $93,3 \%$ de las pacientes obtuvo el alta el mismo día de la cirugía. Una sola paciente presentó un RPM mayor a $100 \mathrm{ml}$, que se resolvió con cateterismo vesical intermitente, logrando el alta médica 22 horas post TOT con RPM de $50 \mathrm{ml}$ (Tabla II). Todas las pacientes presentaron un RPM menor a $10 \mathrm{ml}$ a los 24 meses. Ninguna paciente presentó complicaciones intraoperatorias ni postoperatorias mayores, una sola paciente presentó retención urinaria a las 6 horas de la cirugía. Luego de 24 meses de seguimiento y frente a la encuesta subjetiva de satisfacción para el tratamiento de la IUE, 13 pacientes (87\%) se sienten "sanas", 2 (13\%) "mejor" y ninguna se siente "igual" o "peor"(Tabla III).

\section{DISCUSIÓN}

Los resultados de continencia en esta serie son levemente superiores a los resultados generales publicados en la literatura actual, aunque en muchas de estas publicaciones se considera como resultado exitoso tanto a las pacientes en las que se logró una continencia urinaria normal, como las que han presentado una disminución significativa de su IUE que les permite tener una buena calidad de vida. Si aplicáramos el mismo criterio tendríamos un éxito de $100 \%$ en esta serie $(6,7,8,9)$.

Tabla I

CISTOMETRÍA CLÍNICA (n: 15)

\begin{tabular}{lcccccc}
\hline & $\begin{array}{c}\text { Frecuencia } \\
\text { IUE/Semanal }\end{array}$ & $\begin{array}{c}\text { RPM } \\
(\mathrm{ml})\end{array}$ & $\begin{array}{c}\text { PDM } \\
(\mathrm{ml})\end{array}$ & $\begin{array}{c}\text { CV } \\
(\mathrm{ml})\end{array}$ & $\begin{array}{c}\text { CTV } \\
(+)\end{array}$ & $\begin{array}{c}\text { PTVT } \\
(+)\end{array}$ \\
\hline Promedio & 30 & 6,59 & 180 & 426 & $0 / 15$ & $15 / 15$ \\
Rango & $3-90$ & $0-25$ & $30-360$ & $250-610$ & - \\
\hline
\end{tabular}

IUE: Incontinencia urinaria de esfuerzo. RPM: Residuo postmiccional. PDM: Primer deseo miccional. CV: Capacidad vesical. CTV: Contracciones vesicales. PTVT: Prueba de TVT clínica. 
Tabla II

SEGUIMIENTO POSQUIRÚRGICO (n: 15)

\begin{tabular}{lccccccc}
\hline & 6 horas & 7 días & 3 meses & 6 meses & 12 meses & 18 meses & 24 meses \\
\hline IUE & 0 & 0 & 2 & 0 & 1 & 1 & 1 \\
RU & 1 & 0 & 0 & 0 & 0 & 0 & 0 \\
(RPM $>100 \mathrm{ml})$ & 1 & 0 & 0 & 0 & 0 & 0 & 0 \\
Complicaciones & 1 & & 0
\end{tabular}

RU: Retención urinaria. IUE: Incontinencia urinaria de esfuerzo: RPM: residuo postmiccional.

Tabla III

ENCUESTA SUBJETIVA DE SATISFACCION

\begin{tabular}{lcccc}
\hline & 3 meses & 6 meses & 12 meses & 18 meses \\
\hline Sana & 12 & 12 & 12 & 13 \\
Mejor & 3 & 3 & 3 & 2 \\
Igual & 0 & 0 & 0 & 0 \\
Peor & 0 & 0 & 0 & 0 \\
\hline
\end{tabular}

Nos parece muy importante destacar que en esta serie no se presentó ninguna sobrecorrección (RPM elevado). Actualmente la gran mayoría de los trabajos relacionado con los tratamientos de la IUE evalúan éxito solo con el resultado de continencia urinaria, aunque tal vez muchas de ellas han sido sobre corregidas y en el futuro deberán ser tratadas por esta complicación, generalmente por médicos urólogos, por lo que en muchos casos, el cirujano no estará al tanto de esto, quedando con la sensación de que su tratamiento fue exitoso.

Creemos que los buenos resultados de continencia sin sobrecorrección obtenidos en esta serie, se deben a la rigurosa evaluación preoperatoria e intraoperatoria. En el preoperatorio le otorgamos gran importancia al diagnóstico preciso de la IUE incorporando a la anamnesis, examen ginecológico y la cartilla miccional el estudio cistométrico clínico, junto con la prueba de TVT clínica que permiten: confirmar el diagnóstico de IUE, predecir el resultado de la cirugía, pesquisar patologías vesicales funcionales y pesquisar IUE tipo 3 .

En el intraoperatorio nos parece fundamental el ajuste de la malla ya que la tracción excesiva de las mallas puede sobre corregir la IUE y la tracción deficiente de esta puede subcorregirla, lo anterior, basado en que bajo anestesia raquídea (bloqueo motor del mecanismo fundamental para la continencia de esfuerzo) la condición fisiológica es de escape de orina a presiones muy bajas (sobre 35 $\mathrm{cm} \mathrm{H2O),} \mathrm{y} \mathrm{que} \mathrm{producir} \mathrm{continencia} \mathrm{con} \mathrm{maniobras}$ de valsalva es anormal ya que genera una condición obstructiva, incluso bajo anestesia raquídea en mujeres continentes post cirugía de Burch tienen escape de orina con maniobras de valsalva (3).

En cuanto a la paciente que objetivamente persistió con IUE tenía 49 años, obesa (IMC: 30), con 4 partos vaginales, sin prolapso de órganos pélvicos (POP) y presentaba IUE 70 veces por semana. Luego del TOT disminuyó drásticamente a una vez por semanas y sólo con grandes esfuerzos. En este caso, a pesar de no haber logrado una continencia normal, para ella significó un cambio de vida sustancial lo que se considera exitoso en la mayoría de las publicaciones. Creemos que en ella puede haber jugado un rol importante su índice de masa corporal (IMC) lo que puede haber condicionado a que disminuyera la tensión de la malla permitiendo escapes de orina pero sólo con grandes esfuerzos.

De las dos pacientes que respondieron a la encuesta subjetiva de satisfacción "mejor" y no "sanas", una corresponde a la paciente que objetivamente persistió con IUE y la otra corresponde a una paciente que en el control médico realizado a los 3 meses de seguimiento relató haber presentado escape de orina con esfuerzos en sólo 2 ocasiones sin que se repitiera en el resto de los controles hasta completar 24 meses de observación. Nos llamó la atención una paciente que en los primeros controles se sentía "mejor" y luego en los dos últimos 
controles "sana" pero que nunca presentó IUE, ni complicaciones luego del TOT. Atribuimos esta respuesta a una comprensión poco clara a la pregunta subjetiva durante los primeros controles.

El caso aislado que presentó un RPM de $600 \mathrm{ml}$ el mismo día del TOT, se debió a un retraso en el cateterismo post TOT asociado a una sobre hidratación parenteral, lo que provocó un aumento de la diuresis, sobre distención de la vejiga y posteriormente una vejiga hipotónica transitoria, que con un buen manejo posterior permitió el alta médica a las 22 horas post TOT. Este caso nos ha alertado en que tal vez la utilización de una sonda Foley por 6 horas luego del TOT (hasta que pase completamente el efecto anestésico) y medir el RPM luego de la primera micción espontánea, evitaría la sobre distención vesical por fallas en el cateterismo vesical.

Es muy destacable, aunque la serie sea pequeña, el no haber presentado complicaciones mayores intra ni postoperatoria lo que demuestra que esta técnica es segura cuando existe un buen dominio de esta por parte de los cirujanos y un buen manejo de enfermería, realizado en los controles post operatorios especialmente durante las primeras horas en que la paciente permanece con efectos anestésicos.

Con respecto a la técnica quirúrgica se debe poner énfasis en ubicar la malla en la uretra media y realizar una disección muy acotada (con tijera y guía del gancho con el dedo meñique) de la mucosa vaginal con el objetivo de evitar la movilidad de ésta y fallas posteriores del tratamiento. Por otra parte, nos parece fundamental el ajuste intraoperatorio de la malla, porque de otra forma el TOT se realiza a "ciegas", ya que en la práctica el término "libre de tensión" es interpretado de distinta forma por cada cirujano y responderá de distinta forma en cada paciente de acuerdo al grado de movilidad de la uretra media durante los esfuerzos, esto puede aumentar las posibilidades de una subcorrección o una sobrecorrección de la continencia urinaria. En esta serie se presenta una forma objetiva, fácil y rápida de ajustar la tensión de la malla para disminuir al mínimo el riesgo, tanto de la subcorrección como de la sobrecorrección.

Por último, es por todos sabido, que para obtener un resultado adecuado en el tratamiento de la IUE es de suma importancia hacer un diagnóstico preciso, incluyendo pruebas clínicas diagnósticas, predictivas, objetivas, seguidas de pruebas intraoperatorias que disminuyan al mínimo el riesgo de una subcorrección o sobrecorrección de la IUE, acompañadas de pruebas clínicas que evalúen estas condiciones en el seguimiento posterior.

Se necesitarán estudios prospectivos, con un poder adecuado, y seguimiento a largo plazo, que involucren criterios de diagnóstico, y resultados claros, objetivos y comparables para confirmar lo sugerido por esta serie.

\section{CONCLUSIÓN}

Se presenta una técnica de fácil realización por profesionales entrenados en técnicas uroginecológicas, que cumple con el concepto de mínimamente invasiva, que permite un adecuado control de la sub y sobrecorrección quirúrgica, con buena satisfacción de las pacientes.

\section{REFERENCIAS}

1. Temporal M, Newman D, Deutchman M. Stress urinary incontinence: What matters? The Family Practice Education Network (FPEN) 2004;5:1-10.

2. Haylen B, Ridder D, Freeman R, Swift S, Berghmans $\mathrm{B}$, Lee J, et al. An International Urogynecological Association (IUGA) / International Continence Society (ICS) joint report on the terminology for female pelvic floor dysfunction. Int Urogynecol J 2010;21:5-26.

3. MacMillan G, Yunge P, Daviú A, Riera P, Godoy M. Incontinencia urinaria de esfuerzo (IUE) en la mujer. Contribución a la comprensión de su patogenia y tratamiento. Rev Chil Urol 2003;68:51-4.

4. Petri E, Ashok K. Comparison of late complications of retropubic and transobturator sling in stress urinary incontinence. Int Urogynecol J 2012;23:321-5.

5. Mac Millan G, Godoy R, Riera P, Daviú A, Carmona $C$, Jara D, Yunge P. Cirugía tipo TVT en incontinencia femenina con elementos de bajo costo y parámetro predictivo de éxito. Rev Chil Urol 2003;68: 55-8.

6. Dell'oro A. Cirugía en la incontinencia de orina de esfuerzo: cinta suburetral media de prolene $₫$. Experiencia prospectiva del hospital Dr. Sótero del Río. Rev Chil Urol 2003;68:247-61.

7. Roa J, Roa E, Romero G. Experiencia con sling suburetral libre de tensión transobturador (TOT) en el tratamiento de la incontinencia urinaria femenina. Rev Chil Obstet Ginecol 2004;69:294-300.

8. Olmedo T. Safyre transobturador: técnica mínimamente invasiva con sling reajustable. Rev Chil Urol 2005; 70:20-3.

9. Zhua L, Langa J, Haib N, Wongc F. Comparing vaginal tape and transobturator tape for the treatment of mild and moderate stress incontinence. Int J Gynecol Obstet 2007;99:14-7. 